\title{
X-RAY DIFFRACTION FROM ION IMPLANTED ZONES
}

Satish I. Rao, Charles R. Houska, Department of Materials Engineering, Virginia Polytechnic Institute and State University, Blacksburg, Virginia 2406 I; K. Grabowski and J. Claussen, Naval Research Laboratory, Washington, D.C., 20375; and G. Ice and A. Hahenschuss, X-14 NSLS, Brookhaven National Laboratory, Upton, NY 11973.

\section{ABSTRACT}

Measurements of $x$-ray profiles and diffuse scattering from (111), (100) single crystal Niobium films implanted with Nitmgen to average levels of 5 and 0.5 atomic percent are discussed. Theoretical analysis of the asymmetric profiles are used to determine the strain profile in the implanted films. The measured strain profile results from two factors: (i) depth distribution of implants and knock-on damage and (ii) elastic constraints. Residual elastic strains develop due to the constraints imposed by a sapphire substrate. Comparison of the difraction results with thenretical predictions of TRIM indicates the presence of measurable knock-on damage in the films. Huang and Stokes-Wilson scattering measurements made using synchrotron radiation at the ORNL beamiline, Brookhaven National Laboratory, were used to reveal the identity of defects formed during the knock-on process.

\section{INTRODUCTION}

Primary solutions are, under examination containing single foreign interstitials which re main unclustered in octahedral sites [1] because of the choice of low implantation temperaturest An energetic interstitial atom produces damage related self interstitials; and racancies. Self interstitials and vacancies produced by damage become sagregated, due to enhanced difrusion, into a distrihution of small loops at liquid nitmgen temperature.

Four implantation energies were used to pmduce four overlapping Gaussian distributions of $N$ in a 25nnA $\mathrm{Nb}$ single crystal film deposited onfo a thick (001) sapphire crystal. This gires a nonuniform: concentration when superimposed giving an average of 5 at $\% N$. Variations about the average composition produce related variations in the interplanar spacing. Bragg peaks become broadened into intensity hands with a characteristic fine structure which is used to obtain the distribution of interplanar spacing below the free surface. Difraction theory was dereloped to allow for the rather large effect of static displacement fields about interstitial implants as mell as damage related defects. Kinematic theory was used throughout these developments because of the relatively large distortions encountered in ligh fucnce inn-implantation problems.

We find that the distribution of inferplanar spacings is $15 \%$ larger than that predicted theoretically by. TRIM. This has heen sinww in he rclated to the excess expansinn from rexidual 
knock-on damage. Both implanted $N$ and knock-on defect arrangements can produce a laltice expansion and related compressive stresses. Although some stress relaxation results from field cancellation between interstitial and vacancy lonps, complete cancellation does not occur for small loops due to core effects.

\section{ELASTIC-FREE EXPANSION STRAINS}

Consider the overall implanted znne to be contained within semi rigid walls and that it responds elastically without relaxations associated with plastic deformation. That is, the underlying material does not allow a free expansion of the zone. The strains (and stresses) that develop along the implanted zone can be calculated using the method of strain suppression [2!

In treating the purely elastic problem, the implanted zone is allowed to expand freely as uncoupled slabs of constant concentration " $C_{7}$ " and a thickness $d x_{3}$. Each slab undergoes a free expansion (or contraction) due to the pressure exerted by the implanted atoms and knock-on defects. We know that a concentration " $\mathrm{C}_{1}$ " of one kind of point defect and orientation produces a hulk expansion of the lattice given by [3].

$$
\sum_{i=1}^{3} \epsilon_{i i}=\frac{C_{i}}{V} \frac{\partial V}{\partial C_{i}}
$$

where $E_{n}$ are the diagonal components of the strain tensor, expressed in terms of the cubic coordinate system. Cubic symmetry is maintained by allowing each of the axes of the principal strain to be oriented along mutually perpendicular directions in equal numbers. Isotropy is normally lost when the slabs are joined continuously [4] by the method of strain suppression. The elastic strain profile, expressed in terms of the sample conrdinate system, is directly proportional to the variation of " $\mathrm{C}$ ". Slabs are joined together continuously making the total strain along the $l$ ' and 2' parallel to lise free surface, essentially zero thrnughout the implanted zone.

The normal elastic strain, at an angle $\chi$ to the $x_{x}$ direction, is ohtained from [4].

$$
\dot{E}_{e x}^{\prime}\left(x_{3}\right)=\left\{\dot{E}_{e 11}^{\prime}\left(x_{3}\right)-\dot{\epsilon}_{e, 3,}^{\prime}\left(x_{3}\right)\right\} \sin ^{2} z+\dot{E}_{e .33}\left(x_{3}\right)
$$

or

$$
\dot{\epsilon}_{\mathrm{ex}}\left(x_{3}\right)=\frac{C\left(x_{1}\right)}{3 V} \frac{\partial V}{\partial C}\left\{-f^{\prime \prime} \sin ^{2} z-\Lambda\right)
$$

where

$$
\beta^{+1}=\frac{C_{11}+2 C_{12}}{C_{11}+C_{2 n} \Omega}=1-A
$$

and $\Omega$ rrialcs to the transformation to the sample conrdinate system [4]. The total strain in the implanteri $r$ ac, at tilt angle $z$, includes the free expansion strain and the elastic strain, i.e. 


$$
\epsilon_{1}\left(x_{3}\right)=\frac{C\left(x_{3}\right)}{3 V} \frac{\partial V}{d C} r^{\prime \prime} \cos ^{2} x
$$

The measured strain gnes to zern linearly with a $\cos ^{2} z$ dependence, at $z=90^{\circ}$. The ratio of the total strain at $\chi=0^{\circ}$, to the free expansion strain is $\beta^{-1}$ which is a magnification factor that can be as large as 2-3. At an angle $\chi=\chi_{0}$, the total strain is identically equal to the free expansion i.e.

$$
\cos ^{2} x_{0}=1 / \beta^{e-1} \text { or } \sin ^{2} x_{0}=-A_{1} / \beta^{e t}
$$

The main features of the strain equations are illustrated in Fig. I for one element.

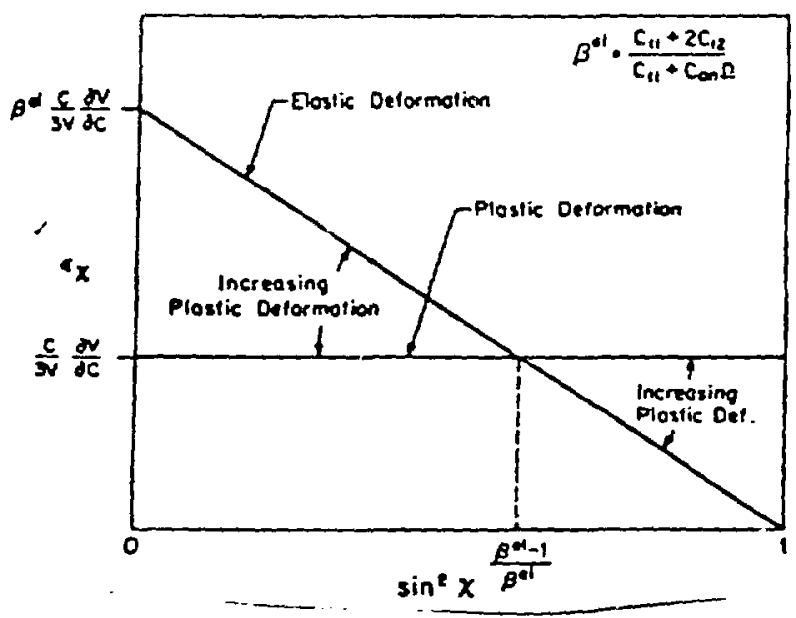

FIG. 1. Plots of Eq. (O) with and without $\beta^{\text {et }}$ expansion factor i.e completely elastic or completely relaxed by plastic deformation $\left(\beta^{e 1}=1\right)$.

A measurement of the strain prorile at various tilt angles $\chi$, allows one to separate the free expansion gradient from the elastic strain gradient. $X$-ray intensity band analysis is used to determine the strain profile and is discussed in a later paper.

If the concentrations are sufficiently small, these results can be applied to more than one type of defect by summing over all defect species, " $\mathrm{j}$ " to obtain the free expansion profile. The profile ohtained when the sample is elastically constrained is ohtained by simply multiplying by $\beta$.

\section{INTENSITY DISTRIBUTION FROM ELASTICALLY CONSTRAINED ZONE}

If the implanted zone is completely constrained by the underlying suhstrate, then the displacement components along $a_{1}$ and $a_{2}$ directions, $X_{m}$ and $Y_{m}$, are identically equal to zero. Therefore, the strains $\epsilon_{i j}$, is only a function of $x_{3}$. By introducing these simplifications, and summing over each layer, one obtains

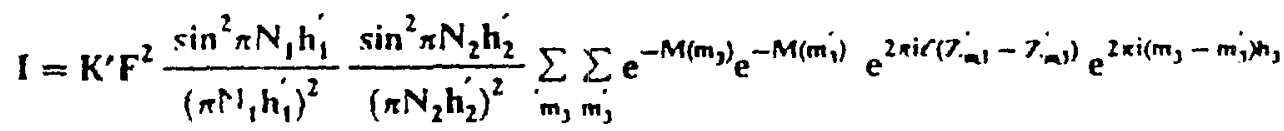

where $K^{\prime}$ is constant over one intensity hand, $F$ is the structure factor for the nomal unit cell. $N_{1} a_{1}$ and $V_{:}:_{2}$ are the dimensions of the crystal alnng $a_{1}$ and $a_{2}$ directions parallel to the free 
surface and $h_{i}=\left(h_{1}-h_{3}\right), h_{2}=\left(h_{2}-k\right)$ are variables in reciprocal space. The exponentials containing $M\left(m_{3}\right)$ and $M\left(m_{3}\right)$ are static attenuation factors determined by the implants and loops [1,5]. If $N_{1} a_{1}$ and $N_{2} a_{2}$ are taken to be large, the intensity in reciprocal space is spread only along the $h_{3}$ direction, and is a product of delta functions with respect to the $h_{1}$ and $h_{2}$ dependence. The variation of the intensity along the $h_{3}$ direction is obtained by numerically summing Eq. (T) over the entire implanted zone.

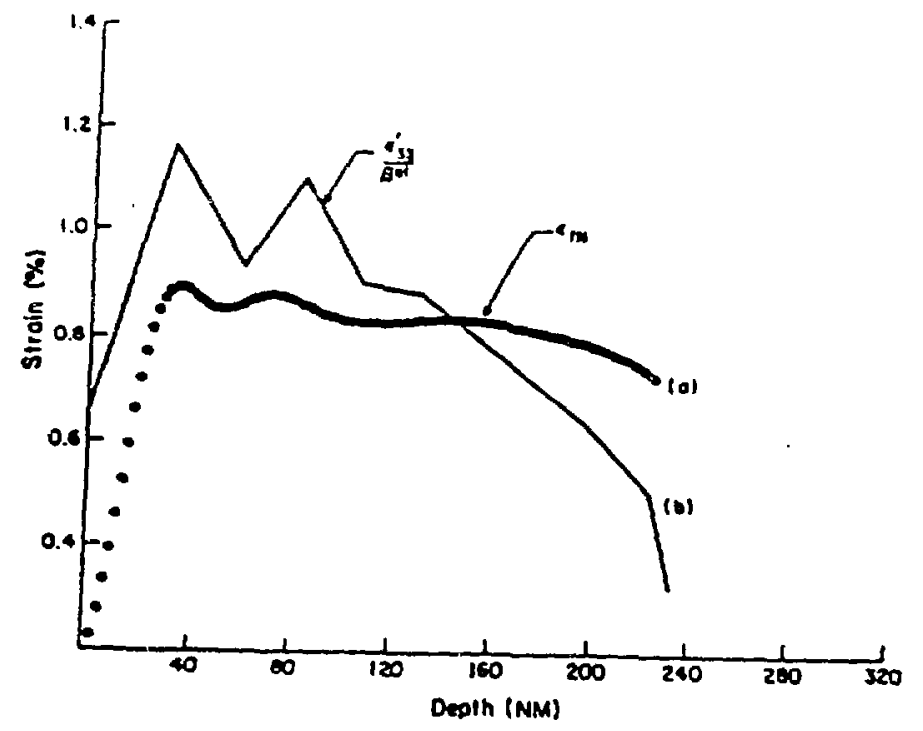

FIG. 2. Free expansion strain profiles for multiply implanted $N$ into a $2500 \mathrm{~A}$ (111) oriented $\mathrm{Nb}$ film on (001) sapphire. Determined from (a) TRIM simulations (b) $x$-ray intensity band analysis.

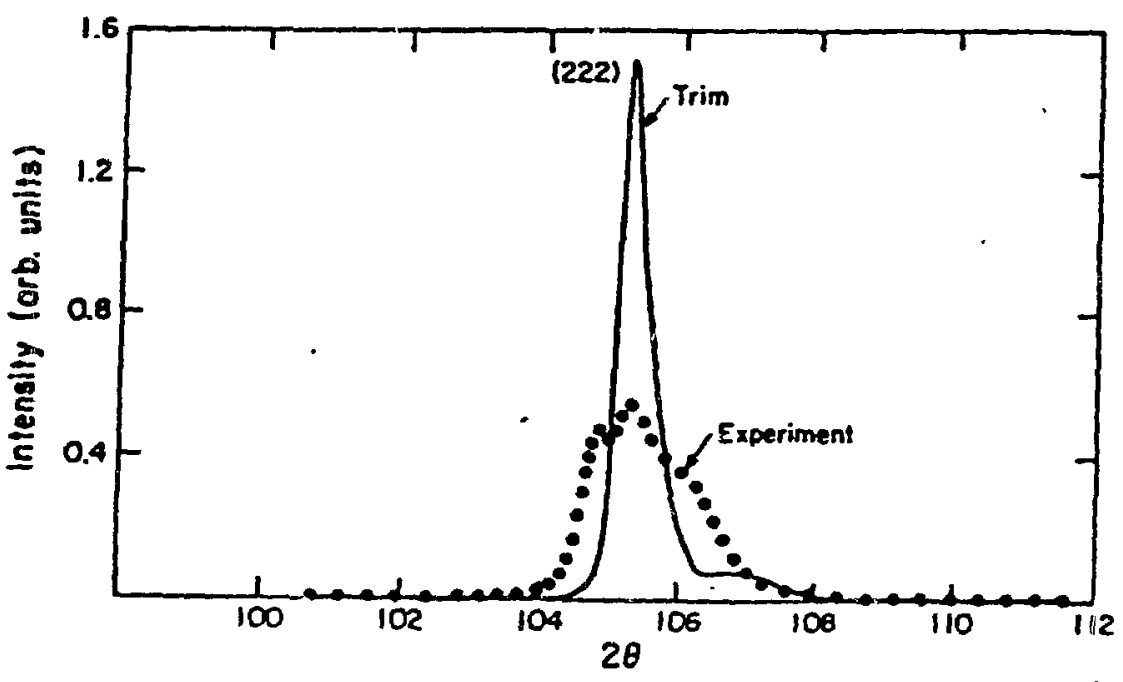

FIG. 3 .

(222) Intensity band from TRIM obtained with curve in Fig. 2(a) using Eq. (7).

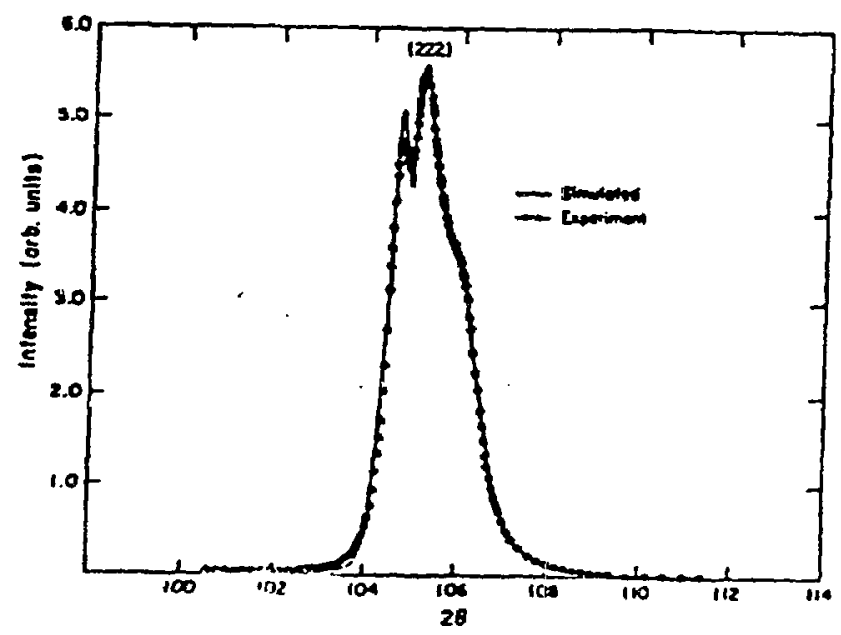

FIG. 4. Experimental (222) intensity band compared with the intensity profile ohtained with the curve in Fig. 2(b) using E.g. (7). 
Figure 2(b) shows an experimentally determined strain profile along the multiply implanted Nh film and the division of such a zone into connecting linear regions. The assumption of linear gradients introduces sawtooth regions in the strain profile when the strain gradient changes sign. A sinusoidal representation of the strain profile would eliminate the sawtooth discrntinuities. However, under the present conditions, the end results from either analysis are not significantly altered.

Figure 3 illustrates the (222) intensity band from strain profile Fig. 2a obtained from TRIM calculations. Clearly, this does not fit. Also, TRIM calculations do not account for the Nb sapphire interface. Figure 4 illustrates our hest fit to the experimental intensity band and gives the experimental curve in Fig. $2(a)$. The $x$-ray analysis shows a strain profile that contains additional detail near the surface and appears to go to zero near the interface. The TRIM cat culation should not be considered beyond $160 \mathrm{~nm}$. Howrever, the peak location is in agreement with the constrained elastic model.

A preliminary examination of a (100) $\mathrm{Nb}$ fim implanted under the same conditions as the (111) orientation gives an intensity band which differs from the results shown with a (111) film. However, the results indicate that they are in accord with an elastically contrained model.

The (222) planes are parallel to the free surface so that the data are obtained from a radial scan. If this is transformed to (001), bands of the type hk $\ell$ must be projected. At an angle of inclination $\chi$ corresponding to the (hk $\ell$ ) band, the projection is given by

$$
\cos x=\frac{l}{\sqrt{h^{2}+k^{2}+l^{2}}}
$$

When this holds, the spread of the intensity along an (hk $\Omega$ ) band in the "hs" direction is identical to the distribution around the corresponding (00/) point. A detailed study of six intensity bands was used to determine the strain distribution along the implanted rone and assess whether the strains along the $I^{\prime}, 2$ directions are zero.

If particle size hroadening is negligible, one can show that the strain broadening is proportional to $\ell[6]$ and one can inter-relate (hk $\left.c^{z}\right)$ distributions to the (001) distribution. The spread in radians is proportional to the overall strain and described by

$$
\Delta 20(\mathrm{rad})=2\left(e \cos ^{2} x\right) \tan \theta
$$

This gives the usual tan0 relation between a shift in d-spacing and the related angular change. The additinna! $\cos ^{2} \chi$ allows for the projection along an angle $\chi$. If this dependence is obeyed, then the purely elastic model is rerified and particle size broadening is negligihle. These data are examined in detail in a later paper.

\section{LATTICE DAMAGE}

$A(111)$ Nh film was implanted to an average composition of $0.5 \% \mathrm{~N}$ at LNT to reduce the d-spacing liu, scuing and thereby allow diffuse scalfering data to he collected. We liave charac- 
terized the knock-on defect structures with Hunng-Stokes-Wilson $x$-ray diffuse scattering using Larson flots [7]. This required 2 difference to he taken betreen the data from implanted and unimplanted single crystal Nb films. Statistically meaningful data could only be obtained with synchrotron radiation. This diffuse scattering, resulting from long range elastic displacement fields, and can be explained by vacancy and self interstitial loops of like size distributions with radii extending from $5-15 \AA$.

The displacement field from knock-on damage bas been identified as loops on (211) planes using an anisolmpic calculation by Ohr [8]. Self interstitial and vacancy loops have like displacement fields except for a change in sign l.e interstitial loops give a positive displacement along the loop axis while vacancy loops give negative values. Although this cancels some of the displacement field, the cores continue to make a contribution and account for the $15 \%$ difference between the $x$-ray results and TRIM in the range from 0 to $140 \mathrm{~nm}$ (Fig 2).

At $0.5 \% \mathrm{~N}$, most of the expansion is due to lattice damage and this is close to the level found at 5\%. Someplace in between these concentrations, the lattice damage appears to saturate causing the lattice disturbance from an increasing number of interstitial $\mathbf{N}$ defects to dominate.

Simulations using expanded rersions of TRIM are capable of giving the distribution of energy loss due to knock-on collisions. We were encouraged to find that this follows the same general form as the excess in d-spacing which is attributed to residual knock-on damage.

\section{CONCLUSIONS}

1. For a 5 at \% implantation of $\mathrm{N}$ in $\mathrm{Nb}$ at LNT, the dominant elastic strain is dive to single $\mathbf{N}$ interstitials.

2. Ay a lower level of $(0.50$ at $\% \mathrm{~N}$ ) lnock-on lattice damage is suficiently large and differem from wative to single interstitials to be measureable using Huang-Stokes-Wilson diffuse scattering from a single crystal filmacontaining a low lewer of $\mathrm{N}$ daon $(0.50$ at q).

3. X-ray intensity hand analysis provides a means of determining the strain profile along an implanted zone.

4. The strains remain elastic without observable plastic deformation.

\section{ACKNOWLEDGEMENTS}

We wnu?d like to acknowledge Professor D. Farkas for providing simulations of the energy deposition curres and the Office of Nayal Research for sponsoring this research on Grant \# N0004-83-K-0750, P00004. Research was performed in part at th. Oak Ridge National Laboratory Bram!ine X-14 at the National Synchrotron Light Snurce, Bronkhaven National Labora- 
tory, sponsored by the Division of Materials Science and Division of Chemical Sciences, US. Department of Energy and under Contract DE-AC05-840R21400 with the Martin Marietta Enerpy Systems, Inc.

\section{REFERENCES}

1. S.I. Rao, E.o. Savino and C.R. Houska in 'Mat. Res. Soc. Symp. Proc.', V82, p187 (1987).

2. S. Timoshenko and J.N. Goodier, Theory of Elasticity (McGraw-Hill, N.Y., 1970).

3. P.H. Dederichs, J. Phys. F. 3,471 (1973).

4. S.I. Rao and C.R. Houskz, J. Appl. Phys. 52, 6322 (1981).

5. M.A. Krivoglaz, Theory of X-Ray and Thermal Neutron Scattering by Real Crystals (Plenum, New York, 1969).

6. C.R. Houska, J. Appl. Phys. 41, 69 (1970).

7. B.C. Larson and F.W. Young, Jr. in 'Point Defects and Defect Interaction in Metals' edited by Jin Ich Takamura, Masio Dojama and Michio Kittani, Unirersity of Tokyo Press, p. 679 (1982).

8. S.M. Ohr, Thys. Stat. Sol. 64, 317 (1974).

\section{DISCLAIMER}

\footnotetext{
This report was prepared as an account of work sponsored by an agency of the United States Government. Neither the United States Government nor any agency thereof, nor any of their bility for the accuracy. completeness, or us implied, or assumes any legal liability or responsiprocess disclosed, or represents that its usefulness of any information, apparatus, product, or ence herein to any specific commercial product, process, or privately owned rights. Refermanufacturer, or otherwise does not necessarily constitute orvice by trade name, trademark, mendation, or favoring by the United States Constitute or imply its endorsement, recomand opinions of authors expressed herein do Government or any agency thereof. The views United States Government or any agency thereor
} 